\title{
ARTICLE
}

\section{Spectral conditions in surveillance specimen container of Czech VVER-1000 reactors}

\author{
Milan Marek*, Milan Brumovský and Stanislav Vandlík \\ Centrum Výzkumu Řež s.r.o (Research Centre Rez), 25068 Husinec-Rez 130, Czech Republic
}

\begin{abstract}
Irradiation conditions of material specimens in containers placed on the internal surface of the reactor pressure vessel that are used for the evaluation of material changes during the lifetime of the reactor should correspond to those which the reactor pressure vessel is exposed to. The Czech Modified Surveillance Program for VVER-1000/V-320 C type of reactor vessels in Temelin NPP specifies the following principles that must be applied: (1) the locations of containers should well monitor the conditions at the beltline region of the reactor pressure vessel (RPV) wall, i.e. the specimen temperatures should be as close to the real ones as possible and the lead factor should be less than 5, (2) the whole set of the specimens for one testing curve should be put in identical neutron fluence conditions, (3) the sets of the specimens should be located at positions of similar/near neutron fluence for the behavior of different materials to be comparable, (4) the withdrawal scheme of the containers should assure monitoring the pressure vessel material as well as neutron fluence during the whole RPV lifetime. For the assessment of the influence of neutron fluence on the change of the material characteristics the integral neutron fluence with energy above $0.5 \mathrm{MeV}$ is used. Spectral index SI05 defined as the ratio of the neutron flux with energy above $0.5 \mathrm{MeV}$ to the neutron flux with energy above $3 \mathrm{MeV}$ is used as the measure of the neutron spectrum change. The article presents the analysis of the spectral index SI05 in the specimens and in the reactor pressure vessel at the beltline region. Special interest was focused on the region under the beltline weld up to the depth equal to one tenth of the thickness $(0.1 \mathrm{~T})$ of the pressure vessel as this depth of the postulated defect is used in the calculations according to the VERLIFE methodology.
\end{abstract}

\section{Keywords: reactor pressure vessel; VVER-1000; spectral index; surveillance specimen}

\section{Introduction}

Irradiation conditions of material specimens in containers placed on the internal surface of the reactor pressure vessel that are used for the evaluation of material changes during the lifetime of the reactor should correspond to those which the reactor pressure vessel is exposed to. The Czech Modified Surveillance Program for VVER-1000/V-320 C type of reactor vessels in Temelin NPP was designed in accordance with the VERLIFE procedure [1]. It specifies the following principles that must be applied:

(1) the locations of containers should well monitor the conditions at the beltline region of the reactor pressure vessel (RPV) wall, i.e. the specimen temperatures should be as close to the real ones as possible and the lead factor should be less than 5 ,

(2) the whole set of the specimens for one testing curve should be put in an identical neutron fluence conditions,

(3) the sets of the specimens should be located at positions of similar/near neutron fluence for the

*Corresponding author. Email: Milan.Marek@cvrez.cz behavior of different materials to be comparable,

(4) the withdrawal scheme of the containers should assure monitoring the pressure vessel material as well as neutron fluence during the whole RPV lifetime.

The containers with the specimens are located symmetrically in positions of maximum neutron fluence on the vessel wall surface, i.e. in the hexagon corner positions (Figure 1). Six containers are designed for the study of the effect of radiation embrittlement, two for the effect of potential annealing and re-embrittlement effects, if necessary. The containers are fixed in special holders that are welded on the inner surface of the RPV wall approximately $400 \mathrm{~mm}$ below the centerline of the beltline region. The design of the container holders allows installing additional containers instead of those already withdrawn.

The containers have inner dimensions $200 \times 300 \times 25$ $\mathrm{mm}^{3}$ (Figure 2) and the specimens are arranged in two layers inside (Figure 3). Each container is equipped with neutron monitors assuring the determination of neutron fluence to each of the test specimens. The containers also contain spectrometric sets of monitors for the determination of neutron spectrum in several positions inside. 


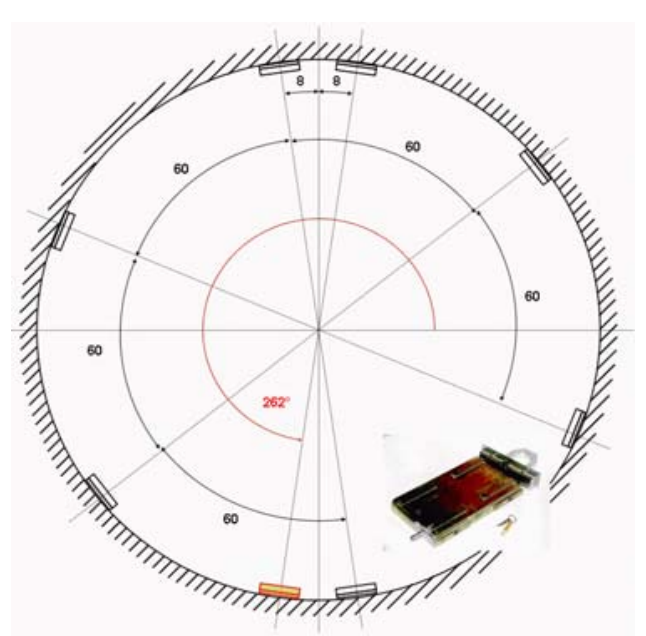

Figure 1. Location of the containers with specimens in the reactor vessel.

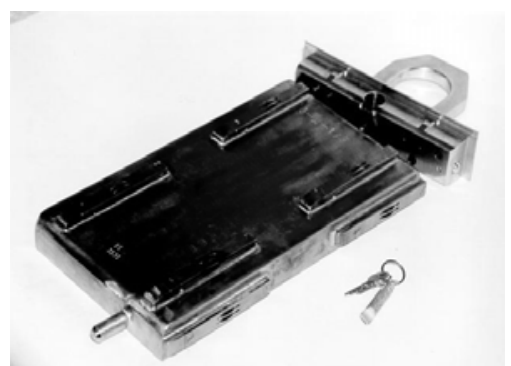

Figure 2. Container of Modified Surveillance Program in VVER-1000 Temelin NPP.

\section{Materials}

The position of the container in the reactor vessel, its construction and the arrangement of the specimens in it have to ensure the neutronic conditions which are as close as possible to those at the reactor pressure vessel (RPV) wall. Especially, good agreement is needed for the neutron spectrum over the irradiated specimens and in the region under the beltline weld up to the depth equal to one tenth of the pressure vessel as this depth of the postulated defect is used in the calculations according to the VERLIFE methodology.

The fast neutron fluence in the surveillance containers and in the reactor pressure vessel (RPV) of VVER-1000 units for the surveillance program is evaluated in accordance with recommendations of US NRC [2]. The calculations are performed using TORT 3D code [3] with 56 group ELXSIR cross section library [4]. The calculation results are adjusted with the LEPRICON code [5] to the activities measured by neutron activation dosimetry both in the surveillance specimen containers and on the outer surface of the RPV.

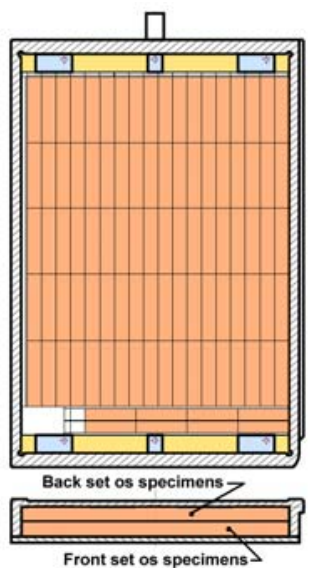

Figure 3. Schematic view of arrangement of surveillance specimens in container.

For the assessment of the influence of neutron fluence on the change of the material characteristics the integral neutron fluence with energy above $0.5 \mathrm{MeV}$ and DPA are usually used. Spectral index $\mathrm{SI}_{05}$ defined as the ratio of the neutron flux with energy above $0.5 \mathrm{MeV}$ to the neutron flux with energy above $3 \mathrm{MeV}$ is used as the measure of the neutron spectrum change.

$$
\mathrm{SI}_{0.5}=\mathrm{F}_{0.5 \mathrm{MeV}} / \mathrm{F}_{3.0 \mathrm{MeV}}
$$

$\mathrm{F}_{0.5 \mathrm{MeV}}$ is integral fast neutron flux related to the neutron energy range above $0.5 \mathrm{MeV}$,

$\mathrm{F}_{3.0 \mathrm{MeV}}$ is integral fast neutron flux related to the neutron energy range above $3 \mathrm{MeV}$.

As one value cannot completely characterize the neutron spectrum, the spectral index $\mathrm{SI}_{05}$ can be used as the measure of the neutron spectrum change.

An estimation of the calculation result uncertainties is based on the comparison of calculated activities with neutron monitor activity measurement that are routinely performed on the outer surface of RPV and in the containers containing the specimens. The uncertainty (1 STD) of the neutron flux $\mathrm{F}_{0.5 \mathrm{MeV}}$ is $\sim 9 \%$, for $\mathrm{F}_{3.0 \mathrm{MeV}}$ is $\sim 13 \%$, for $\mathrm{SI}_{05}$ is $\sim 16 \%$ and for DPA is $\sim 9 \%$.

The calculation model of the neutron transport from the reactor core through the baffle and barrel to the reactor pressure vessel utilizes the $60^{\circ}$ symmetry of the VVER-1000 reactors. Both the axial and horizontal cross section of the reactor are shown in Figure $\mathbf{4}$ and Figure 5.

The container with the specimens that was used for the spectral analyses was positioned at

Radius: $\quad<1996.5,2033.5>\mathrm{mm}$

Height: $\quad<1140,1497>\mathrm{mm}$ above the beltline weld No.3

Angle: $\quad<259.15^{\circ}, 264.85^{\circ}>$.

The radial distribution of fast neutron fluence and DPA were evaluated at the angle of $262^{\circ}$ and at the height that corresponds to the centre of the container (1318.5 mm above beltline weld No.3).

The second radial distribution, that characterizes unperturbed fast neutron flux distribution incident on the 
inner surface of RPV, is calculated at the height corresponding to the half of the core height.

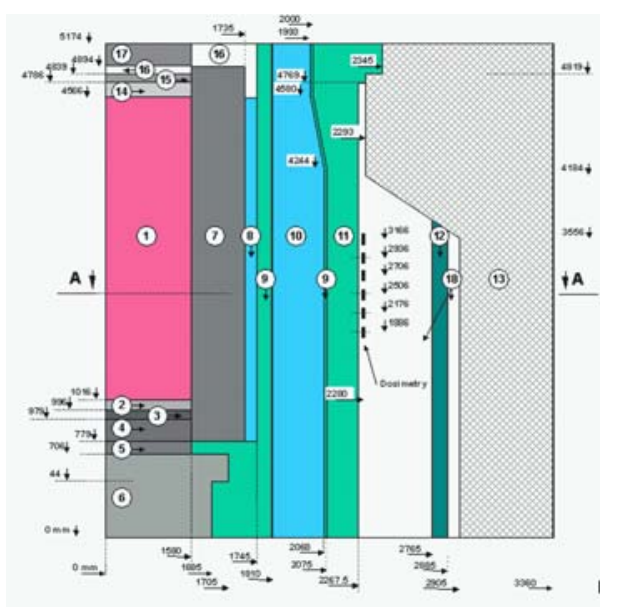

Figure 4. Axial cross section of VVER-1000 model. Legend: 1 - core, 2-4 - nonfuel parts of fuel assemblies, 5-6 - supporting plate, 7 - baffle,, 8,10,16 - water, 9 -barrel, cladding on RPV, 11 - RPV, 12 - insulation, 13 - concrete, 14-15,17 - nonfuel parts of fuel assemblies, 18 - air.

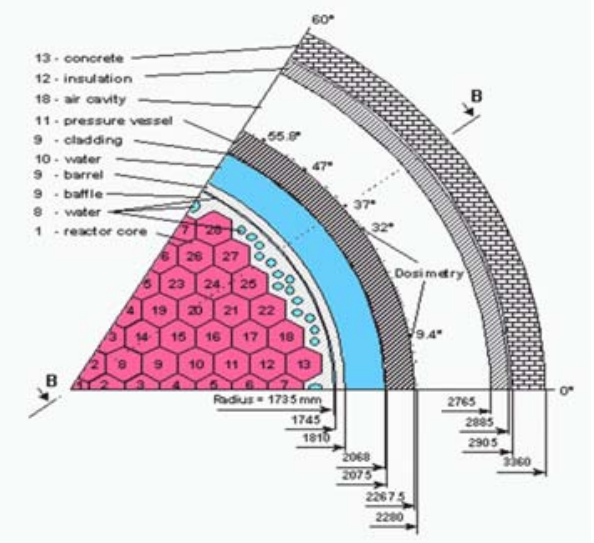

Figure 5. Horizontal cross section of VVER-1000 model (symmetry sector of $60^{\circ}$ ).

\section{Results and discussion}

Calculated fast neutron flux $(\mathrm{E}>0.5 \mathrm{MeV})$ and DPA are shown at Figure 6 and Figure 7, respectively. The container creates a kind of a "shield" for the RPV. That is why the neutron conditions at the RPV surface just behind it are different from the unperturbed by the container (at the half of the reactor core height). Additional $\sim 40 \mathrm{~mm}$ of the steel displacing water has no reasonable influence on the magnitude of the integral neutron flux above $0.5 \mathrm{MeV}$ nor on its distributions in the RPV (Figure 6). On the other hand the fast neutrons are influenced much higher which can be visible on radial distribution of DPA (Figure 7) and spectral index $\mathrm{SI}_{05}$.

The radial change of neutron spectrum characterized by the spectral index $\mathrm{SI}_{05}$ with and without consideration of the container is presented in the Figure 8. The $\mathrm{SI}_{05}$ changes through the centre of the container from 6.67 to 6.96 for the layer of the specimens that are closer to the reactor core while for the layer of specimens oriented towards the RPV it has the value of 7.33. The spectral index in the RPV behind the container increases from 7.62 (surface of the beltline weld) to 10.0 (at the depth of $0.1 \mathrm{RPV}$ thickness). The reason is that the inelastic cross section of $\mathrm{Fe}$ transforms the fast neutron with energy above $0.7 \mathrm{MeV}$ to the energy region bellow this value.

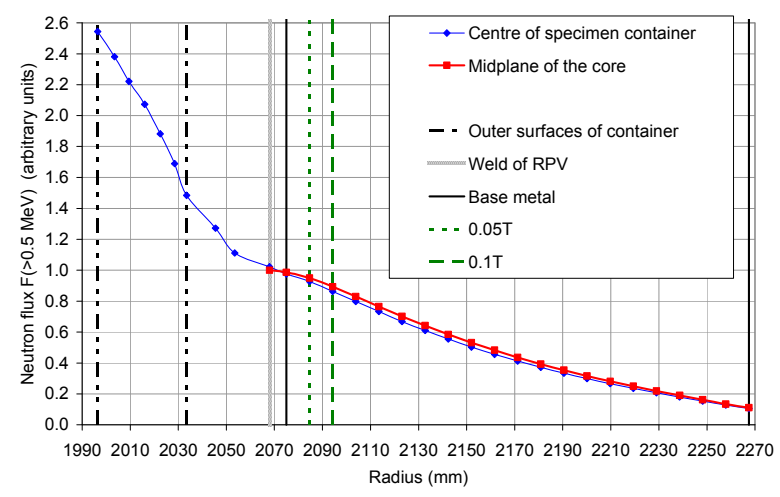

Figure 6. Comparison of radial distribution of fast neutron flux $(\mathrm{E}>0.5 \mathrm{MeV})$ in height corresponding to centre of container and in midplane of core.

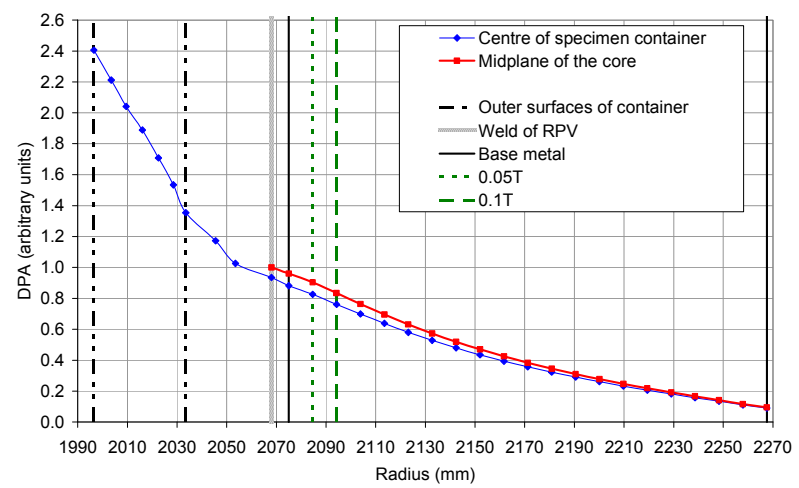

Figure 7. Comparison of radial distribution of DPA in height corresponding to centre of container and in the beltline region.

In the level corresponding to the half of height of the reactor core the $\mathrm{SI}_{05}$ reaches the following values:

- 5.07 on the internal surface of RPV ,

- 5.66 at the border between the beltline weld and base material,

- 6.29 at the depth of 0.05 RPV thickness $(0.05 \mathrm{~T})$,

- 6.97 at the 0.1 thickness of the pressure vessel (this depth of the postulated defect is used in the calculations according to the VERLIFE methodology) $(0.1 \mathrm{~T})$.

The results show good agreement of neutron spectrum in the container (6.67-6.97) and in RPV at the $0.1 \mathrm{~T}$ (6.97) and 0.05T (6.29) depths. The differences of $\mathrm{SI}_{05}$ don't exceed the calculation uncertainties. 


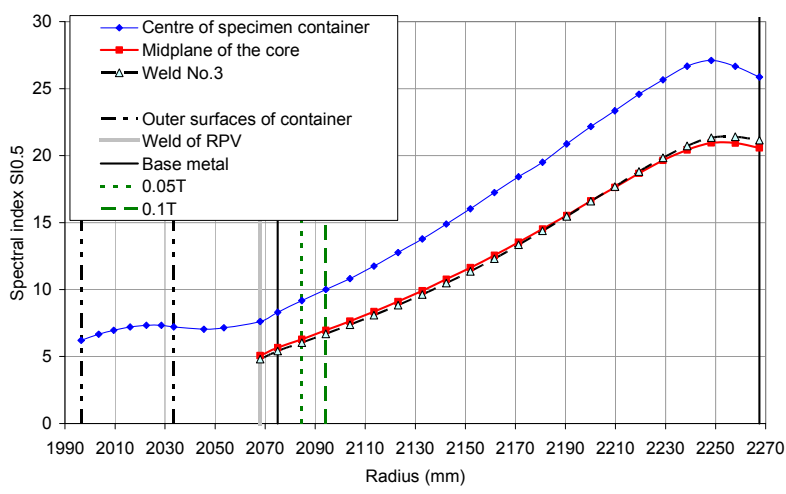

Figure 8. Comparison of radial distribution of spectral index $\mathrm{SI}_{0.5}$ in the height corresponding to the centre of container, in the half of the core and in the height of the beltline weld No.3.

The more detailed changes of the neutron spectrum over the surveillance specimens in the container are shown in the Figure 9. It presents the distribution of spectral index $\mathrm{SI}_{0.5}$ along the height of the container averaged over the front and back layer of specimens. The values are compared with the unperturbed spectral indexes at the inner surface of RPV (beltline 'Weld of RPV'), at the border between the weld and the base metal of RPV ('Base metal'), at the depth of 0.05 thickness of RPV, and at the depth of 0.1 thickness of RPV. The spectral index changes over the specimens in vertical direction from 6.36 to 7.33 . This means that difference in spectral characteristics over all the specimens in the container and the value in 0.1 thickness of RPV (6.97) ranges in $<-9 \%,+5 \%>$. For the depth of 0.05 thickness of RPV (6.29) the range is $<+1 \%$, $+16 \%>$.

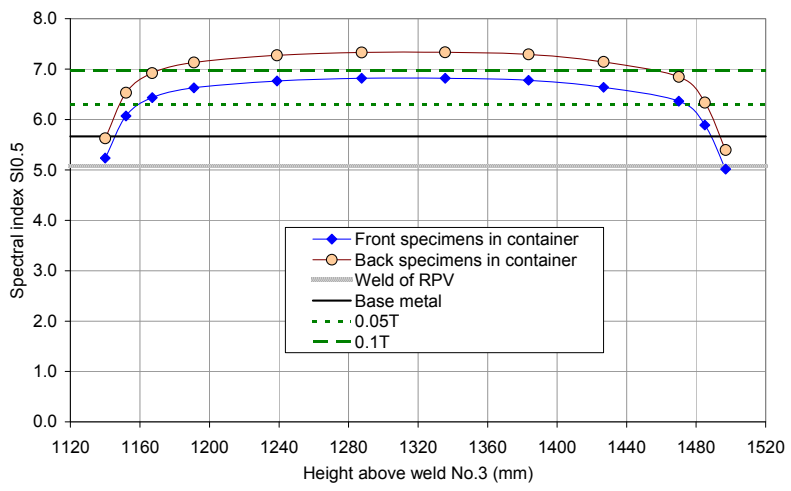

Figure 9. Distribution of spectral index $\mathrm{SI}_{0.5}$ along height of container averaged over front and back layer of specimens.

Figure 10 presents comparison of the radial distribution of $\mathrm{DPA} / \mathrm{F}_{0.5}$ ratio in the height corresponding to the centre of container and in the half of the core height. The average value of the ratio over the front sample layer in the container is $9.01 \mathrm{E}-30 \mathrm{~m}^{2}$ in comparison with $8.85 \mathrm{E}-30 \mathrm{~m}^{2}$ for the back layer. The values correspond well with the ratios on the inner surface of $9.76 \mathrm{E}-30 \mathrm{~m}^{2}$, in the border between beltline weld and base material of $9.50 \mathrm{E}-30 \mathrm{~m}^{2}$, at the depth
$0.05 \mathrm{~T}$ of $9.28 \mathrm{E}-30 \mathrm{~m}^{2}$, and $0.1 \mathrm{~T}$ of $9.11 \mathrm{E}-30 \mathrm{~m}^{2}$ as well. The differences of the ratios are better than $10 \%$ even for the specimens $\left(9.01 \mathrm{E}-30 \mathrm{~m}^{2}\right)$ and for the internal surface of RPV $\left(9.50 \mathrm{E}-30 \mathrm{~m}^{2}\right)$. The ratios for $0.05 \mathrm{~T}$ and $0.1 \mathrm{~T}$ do not differ more than $5 \%, 3 \%$ from the value for specimens. Thus, as this ratio $\mathrm{DPA} / \mathrm{F}_{0.5}$ is practically constant through the whole RPV thickness, the choice of neutron threshold energy for fluence characterization in VVER reactors is well supported. Both the fast neutron fluence with energy above $0.5 \mathrm{MeV}$ and DPA can be used for the characterization of material changes.

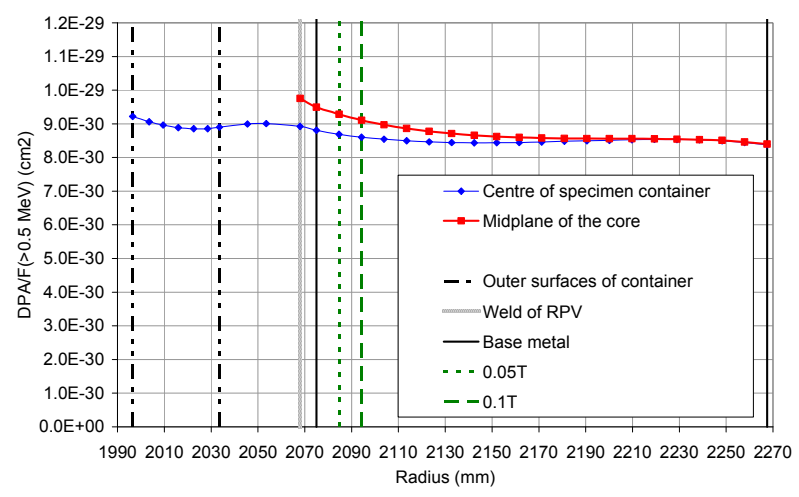

Figure 10. Comparison of radial distribution of DPA/ $\mathrm{F}_{0.5 \mathrm{MeV}}$ ratio in height corresponding to centre of container and in the half of the core height.

\section{Conclusions}

The design of the surveillance container of the VVER-1000 unit at NPP Temelin ensures the irradiation conditions corresponding to those in the region under the weld of RPV beltline up to the depth of 0.1 thickness what is the depth of the postulated defect used in the calculations according to the VERLIFE methodology.

The results of the calculations show that the spectral indexes $\mathrm{SI}_{0.5}$ in the container differ from the value at the depth $0.1 \mathrm{~T}$ RPV by $<-9 \%,+5 \%>$. The neutron spectrum at the depth $0.05 \mathrm{~T}$ is slightly softer then spectrum over the specimens in the container. Spectral indexes in specimens are by $<+1 \%,+16 \%>$ higher then in $0.05 \mathrm{~T}$ RPV.

The ratio of DPA and the neutron flux with energy above $0.5 \mathrm{MeV}$ over the specimens in the container is practically constant as its variation is $1 \%$ (1STD).

\section{Acknowledgements}

The work was supported by Research Project MSM 2672244501 of Centrum Vyzkumu Rez s.r.o.

\section{References}

[1] M. Brumovsky (ed.), Unified procedure for lifetime assessment of components and piping in VVER NPPs "VERLIFE”, COVERS-WP4-D4.10, EURATOM 2008. 
[2] Calculation and Dosimetry Methods for Determining Pressure Vessel Neutron Fluence, Regulatory Guide 1.190, US NRC March 2001.

[3] W. A. Rhoades and F. R. Mynatt, The TORT/PC Three-Dimensional Discrete Ordinate Neutron/Photon Transport Code, CCC-543, RSIC, March 1994.
[4] The ELXSIR Cross Section Library for LWR Pressure Vessel Irradiation Studies, Update of ENDF/B V Mod-3, 1986.

[5] PSR-277/LEPRICON, PWR Pressure Vessel Surveillance Dosimetry Analysis System, RSIC-ORNL, 1991. 